\title{
Antibody mediated rejection. Evidence based medicine. What is the current evidence?
}

Maria do Mar Menezes, Inês Aires, Fernando Nolasco

Department of Nephrology, Centro Hospitalar Universitário Lisboa Central, Hospital Curry Cabral, Lisbon, Portugal.

\section{ABSTRACT}

Antibody mediated rejection (ABMR) is a major cause of kidney graft loss. The ideal treatment remains unknown, is not standardized and there is little evidence to support the use of any specific therapy. This paper aims to reflect on the current state of this challenging diagnosis, either active or chronic active ABMR, and its treatment options. The current treatments encompass plasma exchange, steroids, intravenous immunoglobulin, rituximab, bortezomib, eculizumab, C1 inhibitors, IL 6 inhibitors and IgG-degrading enzyme of Streptococcus pyogenes among others. We will try to analyze the main studies that sustain or reject the current and future use of these techniques and drugs.

Key words: Antibody mediated rejection, donor-specific antibodies, kidney transplant, microvascular injury, renal allograft failure.

\section{INTRODUCTION}

In recent years, the diagnosis of antibody mediated rejection (ABMR) has been rediscovered, more commonly recognized and is presently considered a major cause of kidney (and other graft) loss.

The ideal treatment of $A B M R$ remains unknown and is not standardized $^{1,2}$. The KDIGO Clinical Practice Guideline for the Care of Kidney Transplant Recipients reports is now outdated, as it reports up to $2009^{3}$. Recently a group of experts has put together a consensus document on ABMR treatment, but concluded that there is so far little evidence to support the use of any specific therapy ${ }^{4}$.

This paper aims to reflect on the current state of this challenging diagnosis, either active or chronic active ABMR, and its treatment options.

Evidence rating scale for therapeutic studies has five levels. The first level of evidence comprises high-quality, multi-centered or single-centered randomized controlled trials (RCT) with adequate power or systematic review of these studies. The second level is based on lesser-quality RCT, prospective cohort study or systematic review of these studies; the third level on retrospective comparative study, case-control study or systematic review of these studies; the fourth level on case series and the fifth on expert opinion or case reports ${ }^{5}$.

Most studies sustaining the treatment options of $A B M R$ derive from case reports, case series or non-RCT, therefore allowing an evidence level 2-4. Equally, the results of the published RCT do not allow strong recommendations in the face of the negative results obtained.

\section{ABMR: from diagnosis to prognosis.}

ABMR is a clinical pathological diagnosis, firstly identified in the beginning of transplant era, called hyperacute rejection, characterized by rapid and irreversible transplant rejection, related to the presence in the recipient of high titers of complement fixing antibodies against HLA antigens present in the donor. The work of Paul Terasaki was essential in identifying methods to detect these antibodies, preventing such irreversible rejections.

Only with the identification of markers of interstitial capillary antibody deposition (C4d immunostaining) was ABMR rediscovered and evolved to meet the current criteria, based on the International Banff classification of kidney allograft rejection (2019) ${ }^{4}$.

Antibody mediated rejection was first recognized within the Banff classification in the early and mid-2000s and has undergone extensive updating and revision since then ${ }^{6}$.

Three major criteria must be satisfied. First the histological evidence of graft injury; secondly the histological evidence of antibody endothelial interactions and thirdly the presence of circulating donor-specific antibodies (DSA) ${ }^{7}$.

Serial revisions have permitted exceptions to these initial criteria, the most significant in 2013 after several studies suggesting C4dnegative $A B M R^{6}$. Therefore, the requirement for $C 4 d$ detection was removed and this category was broadened.

In addition, microarray analysis of endothelial transcripts - a technique that applies a molecular phenotype to allograft tissue using extracted RNA to examine patterns of altered gene expression 


\section{Table 1}

Banff 2019 classification of active ABMR

\begin{tabular}{|c|c|}
\hline Active AMR & All 3 criteria must be met for diagnosis \\
\hline 1 & $\begin{array}{l}\text { Histological evidence of acute tissue injury, including } 1 \text { or more of the following: } \\
\text { (a) Microvascular inflammation ( } \mathrm{g}>0 \text { or ptc }>0 \text { ), in the absence of recurrent or de novo glomerulonephritis. In the presence of acute T-cell-mediated rejec- } \\
\text { tion, borderline infiltrate, or infection, ptc }>=1 \text { alone is not sufficient and } g \text { must be }>=1 \text {. } \\
\text { (b) Intimal or transmural arteritis }(v>0) \\
\text { (c) Acute thrombotic microangiopathy, in the absence of any other cause (d) Acute tubular injury, in the absence of any other apparent cause }\end{array}$ \\
\hline 2 & $\begin{array}{l}\text { Evidence of current/recent antibody interaction with vascular endothelium, including } 1 \text { or more the following: } \\
\text { (a) Linear C4d staining in peritubular capillaries or medullary vasa recta } \\
\text { (b) At least moderate microvascular inflammation ( }[\mathrm{g}+\mathrm{ptc}] \geq 2 \text { ) in the absence of recurrent or de novo glomerulonephritis, although in the presence of a } \\
\text { T-cell-mediated rejection, borderline infiltrate, or infection, ptc } \geq 2 \text { alone is not sufficient and } \mathrm{g} \text { must be }>1 \text {. } \\
\text { (c) Increased expression of gene transcripts/classifiers in the biopsy tissue strongly associated with AMR, if thoroughly validated. }\end{array}$ \\
\hline 3 & $\begin{array}{l}\text { Serological evidence of donor-specific antibodies (DSA to HLA or other antigens); C4d staining or expression of validated transcripts/classifiers as noted in cri- } \\
\text { terion } 2 \text { may substitute for DSA. }\end{array}$ \\
\hline Chronic active AMR & All 3 criteria must be met for diagnosis \\
\hline 1 & $\begin{array}{l}\text { Morphological evidence of chronic tissue injury, including } 1 \text { or more the following: } \\
\text { (a) Transplant glomerulopathy }(\mathrm{cg}>0) \text { if no evidence of chronic thrombotic microangiopathy or chronic recurrent/de novo glomerulonephritis; includes } \\
\text { changes evident by electron microscopy alone. } \\
\text { (b) Severe peritubular capillary basement membrane multilayering on electron microscopy. } \\
\text { (c) Arterial intimal fibrosis of new onset, excluding other causes }\end{array}$ \\
\hline 2 & Identical to criterion 2 for active ABMR, above \\
\hline 3 & $\begin{array}{l}\text { Identical to criterion } 3 \text { for active ABMR, above. } \\
\text { Biopsies meeting criterion } 1 \text { but not criterion } 2 \text { with current or prior evidence of DSA (post-transplant) may be stated as showing chronic ABMR, however } \\
\text { remote DSA should not be considered for diagnosis of chronic active or active ABMR. }\end{array}$ \\
\hline
\end{tabular}

- provided further evidence for C4d-negative $A B M R^{6}$. See table 1 for more details.

The table shows the Banff 2019 criteria for acute $A B M R^{8}$.

There are several prognostic factors that predict worse outcomes in $\mathrm{ABMR}^{9}$, in the context of $\mathrm{ABO}$ compatible grafts.

Risk of acute rejection may be divided into two periods: at the time of transplant and in the post-transplant period ${ }^{6}$. In the first case, preformed DSA, especially if in high titers, were the main predictors of $A B M R$ and may warrant desensitization ${ }^{10}$.

Main risk factors englobe histologic and serologic features ${ }^{9}$.

Histologic features include concurrent acute T-cell-mediated (cellular) rejection (TCMR); microvascular injury and C4d staining; transplant glomerulopathy and the degree of chronic injury such as interstitial fibrosis and tubular atrophy ${ }^{9}$.

Serological features include the presence of DSA.

First, we must differentiate between the presence or absence of DSA. Patients with ABMR but without detectable HLA-DSA represent a distinct, often transient phenotype with superior allograft surviva ${ }^{11}$. Nevertheless, we may be in the presence of non-HLA DSA that are difficult to detect.

The type of DSA (preexisting or de novo) is related to different outcomes. De novo DSA (mainly in nonadherent patients or inadequate medication) are usually associated to worse outcomes than preexisting DSA (provided presensitized patients with very high complement fixing antibodies are excluded) ${ }^{9}$.

Class 1 DSA are linked to early presentation, more acute and rapidly graft dysfunction, and despite being more responsive to treatment are associated with more graft loss. Class 2 DSA goes the opposite way ${ }^{12}$.

DSA subclass (type of dominant IgG) may also recognize distinct phenotypes of injury, with IgG3 subclass associated with more severe and early injury ${ }^{9}$.

Regarding mean fluorescence intensity (MFI), their detection with values over 6000 increase 100 times the risk of developing ABMR, but results vary from laboratory to laboratory ${ }^{9}$.

In addition, the complement-binding capacity of the antibody is also linked to a higher risk of renal allograft loss. According to Loupy and Lefaucheur ${ }^{13}$, several studies have revealed that patients with C1q-binding donor-specific anti-HLA antibodies have an increased risk of antibodymediated rejection and more severe antibody-mediated injury.

In a recent study, the authors analyzed patients with $A B M R$ and their survival according to HLA-DSA and C4d status. Interestingly, the 10 -year allograft survival rates were $54.3 \%$ for patients with positive DSA and C4d and $58.0 \%$ for positive DSA but C4d negative patients and $72.5 \%$ for patients with negative DSA and $C 4 d^{14}$.

Finally, the graft function/dysfunction seems to be directly related with poor outcomes in patients with $\mathrm{ABMR}^{9}$. 
These features may have implications in therapeutic options, namely that if there is a C1q fixing DSA, we could consider the use of eculizumab. On the other hand, if there are no DSA, perhaps the use of PLEX may be questionable.

\section{ABMR treatment rationale}

Although this is a major issue regarding the success of kidney transplantation, there are no current guidelines for the treatment of ABMR. Protocols are not standardized but rely on three main objectives: the removal of antibodies from circulation, blocking their production and stopping their interaction with the endothelium.

The KDIGO recommendations (2009) include one or more of the following alternatives, with or without corticosteroids: plasma exchange (PLEX); intravenous immunoglobulin (IVIG); anti-CD20 antibody and lymphocyte-depleting antibody ${ }^{3}$. Plasmapheresis or high dose IVIG constitute standard of care (SOC) with different add-on treatments per center preference. The level of evidence is low (2C) due to the lack of randomized controlled trials (RCT) with statistical power to compare efficacy and safety.

In 2018 Loupy and Lefaucheur ${ }^{13}$ reflected on the multiple potential targets for preventing or treating antibody-mediated rejection, namely PLEX and immunoadsorption (IA), steroids, antithymocyte globulin, IVIG; bortezomib, eculizumab, C1 inhibitors, tocilizumab and IgGdegrading enzyme of Streptococcus pyogenes among others.

Unfortunately, protocols for the treatment of $A B M R$ are rarely comparable and the available evidence is generally of low quality 4 . Nevertheless, we will try to analyze the main studies that sustain or reject the current and future use of these techniques and drugs.

\section{AVAILABLE EVIDENCE FOR THE TREATMENT OF ACTIVE AND CHRONIC ACTIVE AMR}

\section{PLEX/IVIG}

The biological rational for PLEX/IVIG use considers that anti-HLA antibodies activate complement and interact with Fc receptors and endothelium. Therefore, their removal by PLEX is associated with better outcomes in kidney transplant recipients. Also, IVIG have pleiotropic effects including neutralization of antibodies/cytokines/activated components of complement, effects on B cells, T cells, and Fc receptors ${ }^{4}$.

In cases of preexisting DSA and active ABMR without chronic features, PLEX plus IVIG and corticosteroids should be used. In patients with chronic active AMR, it has not been shown to improve outcomes and increases the infection risk ${ }^{4}$.

In 2012, Roberts D, Jiang S and Chadban S wrote a systematic review titled "The treatment of acute antibody-mediated rejection in kidney transplant recipients". It included five small RCT (median of 12 patients) and seven controlled but nonrandomized studies. Among the RCT, four evaluated isolated PLEX and one immunoadsorption (IA) - this one with benefit. Regarding PLEX, only one study showed overall benefit; one showed no benefit and two RCT suggested a trend to harm. Other controlled but nonrandomized trials favored the use of rituximab and bortezomib. Despite its popularity, no RCT were identified regarding IVIG. In conclusion the evidence supporting treatment was low for PLEX and IA and very low regarding all other therapies ${ }^{15}$.

More recently, in 2018, another updated systematic review and meta-analysis was published ${ }^{16}$. The evidence was again stated as low for most treatment approaches, and the authors found relevant heterogeneity in treatments, definition of $A B M R$, and follow-up. Nevertheless PLEX, IVIG and steroids are recommended as standard-of-care (SOC) for the treatment of acute ABMR, despite the evidence uncertainty ${ }^{4}$. The addition of rituximab showed little or no difference to early graft survival, and the efficacy of bortezomib and complement inhibitors remains unclear ${ }^{16}$.

The therapeutic protocols and dosages are not standardized. IVIG doses in the literature vary between $100 \mathrm{mg} / \mathrm{Kg}$ and $2 \mathrm{~g} / \mathrm{Kg}$ and differ according to PLEX employment ${ }^{2,17,18}$.

A typical regimen includes daily or every other day plasma exchange consisting of 1.5 plasma volume removal with each treatment followed by IVIG $2 \mathrm{~g} / \mathrm{Kg}$, with or without a single dose of rituximab at $3.75 \mathrm{mg} / \mathrm{m2}^{4,6,19}$.

IVIG, in addition to treating ABMR, is also used in combination with PLEX to desensitize individuals with alloantibody prior to transplant. The reduction of anti-HLA antibody levels allowed increased number of transplants with good long-term outcomes ${ }^{19,20}$.

In conclusion, despite PLEX and IVIG being the mainstay of treatment for acute active AMR, the evidence involves mostly case series and poorly controlled randomized trials ${ }^{4,21}$. Therefore, the KDIGO guidelines have a $2 \mathrm{C}$ level recommendation ${ }^{3}$.

\section{Rituximab}

Rituximab is a B cell depleting agent that has been suggested as a treatment option since 2009.There are several case series suggesting the benefit of rituximab for treatment of acute and chronic $A B M R^{4,22,23}$, but evidence for its use is still low regarding the main RCT published so far.

In 2009, Lefaucheur and colleagues compared the efficacy of the combination of PLEX/Ig iv/anti-CD20 versus high dose Ig iv alone in the treatment of $A B M R,{ }^{24}$. Twenty-four patients were divided into two groups (12 each) and the follow-up was 36 months. Group A was treated with $2 \mathrm{gr} / \mathrm{Kg}$ IVIG over 2 days every 3 weeks $x 4$ doses. In group $B$, patients underwent daily PLEX $(n=4)$ followed by low dose IVIG $100 \mathrm{mg} / \mathrm{Kg}$. After the last PLEX, patients received high dose IVIG as above and 2 weekly doses of rituximab $375 \mathrm{mg} / \mathrm{m} 2$. Graft survival at 36 months was $50 \%$ in group $A$ and $91.7 \%$ in group $B(p=0.02)$. Further, the DSA MFI were lower in group $B(p=0.009)$. The main conclusion of this study was that high dose of IGIV alone is inferior to PLEX/IVIG/anti-CD20 as therapy for ABMR. Nevertheless, this design of this study is questionable as the benefit could be attributed to PLEX and not rituximab. 
In 2016, results from the RITUXERAH trial were published and added some new data to existing knowledge [25]. This was a phase III, multicenter double-blind, prospective, placebo-controlled trial that evaluated 38 patients with acute ABMR. They were randomly allocated into two groups, receiving either rituximab $(375 \mathrm{mg} / \mathrm{m} 2)$ or placebo at day 5 . They all received PLEX, IVIG and steroids. In case of insufficient efficacy, an additional infusion of rituximab was allowed, as also PLEX and IVIG (which could be seen as a bias). The composite primary endpoint was graft loss and no improvement in renal function at day 12 . The two groups showed no graft loss at day 12 and the percentage of decrease in serum creatinine was similar with $52.6 \%$ and $57.9 \%,(p=0.744)$. Regarding secondary outcomes at one year, there were no deaths; only one graft loss in each group; no differences in proteinuria or extra administrations of rituximab, PLEX and IVIG, and there was also a trend to better renal function in the rituximab group. The main changes were in the histological features. A significant decrease in microvascular inflammatory score (glomerulitis and peritubular capillaritis) was seen at six months in the rituximab group but with no statistic relevance between groups. On the other hand, there was a substantial increase in chronic injury (interstitial fibrosis and tubular atrophy) in the placebo group at six months $(p=0.015)$. In conclusion, the addition of rituximab to SOC (PLEX, IVIG and steroids) did not improve early outcomes. This study was underpowered (limited number of patients and timeline) and significant differences may have been undetected. However, it continues to be used in general transplant practice.

An extension of this study was performed, to evaluate the 7-year outcomes according to the treatment received (rituximab or placebo) [26]. All 38 patients were included, and the results revealed that eleven patients had received only placebo and 27 patients at least one dose of rituximab (some patients received rituximab "in rescue"). Graft survival, creatinine levels, proteinuria and infectious complications were not statistically different between groups. There was a trend in the rituximab group for a higher incidence of neoplasms, mostly cutaneous cancers.

As a conclusion, the authors found no benefit of rituximab in addition with PLEX, IVIG and steroids, in acute ABMR ${ }^{26}$.

\section{Bortezomib}

Bortezomib is a proteasome inhibitor that targets antibody producing plasma cells.

Several uncontrolled studies have suggested some benefit, within combined treatment with PLEX, IVIG, steroids and antibody depleting agents ${ }^{4}$.

The BORTEJECT trial is the first RCT to investigate the results of bortezomib as a treatment option for $A B M R^{27}$. As we know, late $A B M R$ is a leading cause of allograft failure and therefore this study might cast some light onto this challenging diagnosis. Forty-four patients with acute or chronic ABMR and positive DSA were enrolled with a median time of five years post-transplant. Two groups were randomly assigned to receive either bortezomib $(n=21)$ or placebo $(n=23)$, in two cycles three months apart, with a follow-up of 24 months. The primary endpoint - the eGFR (estimated glomerular filtration rate) slope was not different between groups $-0.5 \mathrm{ml} / \mathrm{min} / 1.73 \mathrm{~m}^{2} /$ year $(\mathrm{p}=0.86)$. At the end of follow-up, overall graft and patient survival, proteinuria, DSA levels and histologic features were also similar between groups. In addition, severe adverse effects (gastrointestinal and hematologic toxicity) were more common in the bortezomib group. This trial failed to demonstrate the benefit of bortezomib in preventing eGFR loss and reduction in DSA, adding severe toxicity and is therefore not recommended, alone, for treating late ABMR. In order to investigate this therapy as an add-on to classical treatment, a new trial is on course (NCT 02201576). The Woodle group is a strong proponent of bortezomib use, frequently in combination with other strategies ${ }^{28}$.

\section{Eculizumab}

Eculizumab is a C5 antibody, therefore blocking terminal complement activation.

There are several case series that analyze the use of eculizumab in renal transplants recipients, mostly in desensitization protocols. In one study, despite decreasing acute clinical ABMR rates, eculizumab treatment did not prevent the development of chronic $A B M R^{29}$. Other cases failed to prove benefit ${ }^{30}$, probably because antibody mediated damage is not restricted to complement dependent mechanisms and thus complement blockade may be ineffective in preventing transplant glomerulopathy (TG) in the future.

Lefaucheur and colleagues performed a prospective study with the aim of identifying the specific biologic effect of complementactivating anti-HLA DSAs in the kidney allograft and to evaluate whether antibody complement-activating capacity influences the response to complement inhibition therapy ${ }^{31}$. The results revealed a specific histomolecular kidney allograft rejection phenotype characterized by complement deposition and accumulation of natural killer cells and monocytes/macrophages in capillaries and increased expression of five genes indicative of endothelial activation, natural killer cell and monocyte/macrophage activation.

The use of eculizumab altered this histomolecular phenotype and was linked with a decreased 3-month rejection incidence rate in patients with complement-activating DSAs $(P=0.001)$ but not in those with noncomplement-activating DSAs $(\mathrm{P}=0.65)^{31}$.

In 2017, a pilot RCT evaluated the efficacy of eculizumab in chronic $A B M R^{32}$. The primary endpoint was the change in eGFR trajectory over the treatment period. Fifteen patients, transplanted for at least six months, with positive DSA (MFI>1100) were enrolled. The treatment group received six months of eculizumab followed by a six-month observation period and the control group was kept under observation only. Despite a better trend in eGFR within the treatment group, there was no significant difference between groups $(p=0.09)$. The small number of patients and the limited follow-up might have contributed to these results.

Finally, a monocentric retrospective study of 14 patients who had received eculizumab for a severe active ABMR was performed ${ }^{33}$. Eight patients did not respond to treatment and univariate analyses showed that the time of occurrence of ABMR and chronic glomerular lesions 
were associated with outcome. Hence, eculizumab was more efficient at treating AMR if it occurred within the first-year post-transplantation, which may reflect the more prominent involvement of the complement cascade in the early versus later occurrence of an $A B M R^{33}$.

\section{IL6 Inhibitors}

The development of de novo DSA remains a major concern in chronic $A B M R$ and contributes to renal allograft loss. Current evidence suggests that the proinflammatory IL6 plays an important role in the development of these de novo DSA and chronic ABMR. Therefore, blocking the interaction between IL6 and its receptor is a strategy that has recently been investigated, to prevent the development of DSA ${ }^{34,35}$.

A unicentric open label case study was undertaken in 36 patients with chronic $A B M R$ and positive DSA who failed standard therapy (IVIG and rituximab+-PLEX) [34]. Protocol mensal infusions of the IL6 inhibitor, tocilizumab, were administrated and patients were monitored for DSA and long-term outcomes. At two years and 5 years there was a significant decrease of DSA and stabilization of renal function $(p=0.043)$. The authors also found important reductions in scores of glomerulitis and peritubular capillaritis $(p=0.0175)$ and $C 4 d$ graft deposition $(p=0.0318)$. At six years, patient and graft survival were $91 \%$ and $77 \%$. No significant adverse effects were recorded. In conclusion, tocilizumab may alter DSA production, providing good long-term outcomes in patients with chronic antibody mediated lesions. In 2019, another study complemented these results, showing reductions of total IgG, IgG1-3 and anti-HLA-total IgG and IgG3 levels, suggesting once again that tocilizumab suppresses IgG production ${ }^{35}$.

Recently, tocilizumab was also evaluated as an add-on therapy for SOC of acute active ABMR in a small single-center observational study of seven patients [36]. The results showed a $50 \%$ or superior reduction in DSA in 4 of 6 patients and DSA stabilization in all other. Additionally, renal function improved or stabilized in all patients ${ }^{36}$.

Based on the promising results, there are currently other IL6 inhibitors (clazakisumab), being evaluated in clinical trials ${ }^{37}$.

\section{C1 inhibitors}

Taking into account the importance of the C1q fixing DSAs, a new therapeutic target emerged to try to prolong protection against complement damage. First, a unicentric phase I/II RCT evaluated 20 hypersensitized patients (panel reactive antibody $>50 \%$, positive DSA and positive flow cytometry crossmatch) to whether treatment with $\mathrm{C} 1$ inhibitor (C1INH) would protect renal grafts from ABMR. They concluded that this treatment was safe and could inhibit complement mediated damage after an incompatible transplant, also reducing $\mathrm{C} 1 \mathrm{q}$ fixing DSA and therefore could be an "add-on" therapy option ${ }^{38}$. Another trial, a multicentric phase II RCT, evaluated safety, tolerability and efficacy of $\mathrm{C} 1 \mathrm{INH}$ in the treatment of acute $\mathrm{ABMR}^{39}$. Eighteen patients with less than 12 months post-transplant were enrolled. The experimental group was given $\mathrm{C} 1 \mathrm{INH}$ as an adjuvant therapy to SOC. The results showed no difference at day 20 and day 90 regarding the evolution of serum creatinine but a post-hoc analysis at six months revealed some better histological features in the treatment arm. No features of TG were found in the C1INH group while TG was diagnosed in three out of seven patients the placebo group. This trial's major limitations are the small sample size and the short follow-up period. A single arm (but also very small) study also showed promising results [40]. Six patients with unresponsive ABMR and acute allograft dysfunction received C1INH and IVIG for six months. The primary endpoint was the change of eGFR and all patients showed improvement $(p=0.0277)$. There was also a significant decrease in $\mathrm{C} 4 \mathrm{~d}$ deposition rate and a change in C1q DSA status. These results conclude that the addition of C1INH to IGIV is safe and may improve graft function.

\section{Imlifidase (IdeS)}

IdeS is an enzyme derived from Streptococcus pyogenes that cleaves all IgG subclasses and neutralizes all IgG in the body within hours. It can therefore rapidly eliminate DSA, making it a promising therapy in desensitization. IdeS has also been studied for the treatment of AMBR via effectively blocking complement dependent cytotoxicity and antibody mediated cellular cytotoxicity 4 .

In one study, IdeS was administrated to 25 hypersensitized patients with an incompatible donor ${ }^{41}$. This therapy reduced or eliminated DSA in 24/25 patients and allowed incompatible transplantation. ABMR later developed in ten patients with good response to treatment. Another small trial was recently published with similar results ${ }^{42}$.

A new RCT is open to evaluate the efficacy and safety of IdeS in treatment of active or chronic active ABMR, comparing to PLEX ${ }^{43}$. The sample will comprise 30 patients and the primary endpoint is the reduction of DSA at day five of treatment and renal function and histological features are among some of the secondary endpoints.

The development of rebound DSA and anti-IdeS antibodies are potential drawbacks of this therapy. Ides will probably be used in highly sensitized patients, allowing patients to be transplanted independently of their crossmatch status but will unlikely be used as an isolated treatment for $\mathrm{ABMR}^{4}$.

\section{Novel/emerging therapies}

Daratumumab is another drug that may play a role in ABMR treatment. CD38+ is a transmembrane protein highly expressed on plasma cells and natural killer cells. In an interesting case report of a patient diagnosed with smoldering myeloma and chronic active ABMR, 13 years after kidney transplantation, the CD38 monoclonal antibody daratumumab was used for a nine-month period with encouraging results [44]. There was a continual CD138+ cell depletion in the bone marrow and blood and the previous DSA anti-HLA class II in serum became undetectable. After three months, the follow-up biopsy had a total resolution of microcirculation inflammation (g+ptc: 3 to 0 ) and there was a stabilization of kidney function ${ }^{44}$. These results may open a new window of possibilities regarding treatment of $A B M R$.

Regarding the major limitations of treatment of chronic active (ca) ABMR, belatacept, a fusion protein CTLA4-Ig was assessed. The authors 
converted 19 patients, with caAMR and a high degree of chronicity at the time of diagnosis, from tacrolimus to belatacept at a median of 44 months post-kidney transplant ${ }^{44}$. The encouraging results in the belatacept group showed progressive improvement in estimated glomerular filtration rate $(p=0.02)$, as compared to a steady decline noted in the controls ${ }^{45}$.

Another interesting approach is the sequential use of a proteasome inhibitor (bortezomib) and the fusion protein CTLA4-Ig (belatacept) [46]. The authors hypothesized that the reversal of acute ABMR would require a rapid elimination of antibody-secreting plasma cells followed by the sustained inhibition of their generation. Six patients with acute ABMR were treated and showed sustained disappearance of DSA for a follow-up of 10-30months. They concluded that due to the complementary mechanism of action, these drugs were able to rapidly reverse DSA responses and prevent rebound ${ }^{46}$. The major limitations are the concomitant use of PLEX/IVIG, the reduced number of patients and a lack of control group. Nevertheless, it is also a new road to investigation.

\section{CONCLUSION}

Despite the body of work that has been done, ABMR remains a noteworthy contributor to renal allograft failure.

It is still a difficult diagnosis and its treatment remains empiric as there is only low-grade evidence for supporting the therapy strategies currently used.

Despite low-grade evidence, PLEX/IVIG remains the cornerstone of ABMR treatment but therapeutic protocols and dosages are not standardized.

Regarding the adjuvants, rituximab is one of the most used drugs, but the main RCT RITUX-ERAH failed to demonstrate long-term benefit.

Despite the theoretical advantages of bortezomib, the BORTEJECT trial has also failed to prove benefit.

On the other hand, eculizumab showed some clinical and histological advantage especially in early ABMR associated with C1q-fixing DSA.

The development of de novo DSA remains a major concern in chronic $A B M R$ and contributes to renal allograft loss. Taking this into account, IL6 emerged with some promising results by altering DSA production.

Also, the $\mathrm{C} 1 \mathrm{q}$ inhibitors, regardless of small RCT, exhibited safety and may improve graft function.

The Imlifidase (IdeS) was assessed and shown to rapidly eliminate preformed DSA, allowing incompatible transplantation. Its role in ABMR treatment is still being investigated.

Finally, novel or emerging therapies such as daratumumab and belatacept have been used in small cohorts with promising results.
Recommended SOC treatment for active ABMR with preexisting DSA includes PLEX, IVIG, and corticosteroids [3]. Adjunctive therapies comprise complement inhibitors and rituximab. On the other hand, the SOC treatment for active ABMR but with de novo DSA, includes optimization of baseline immunosuppression (e.g., add steroids if on a steroid-free regimen) and as adjunctive therapies PLEX, IVIG and rituximab ${ }^{3}$. Regarding chronic ABMR, if we have preexisting DSA, we should optimize baseline immunosuppression and consider IVIG as adjunctive therapy. In case of de novo DSA we can also consider IVIG ${ }^{3}$.

So, even though knowledge of its pathophysiology is increasing, and new weapons are under investigation, the complexity of this subject and the heterogeneity of the phenotypes render trials difficult to design and enroll. Therefore, ABMR remains a challenge for the scientific community.

Disclosure of potential conflicts of interest: none declared

\section{References}

1. Montgomery RA, Loupy A and Segev DL. Antibody-mediated rejection: new approaches in prevention and management. Am J Transplant 2018 Jan;18(3):3-17.

2. Comai G, Ravaioli M, Baraldi $\mathrm{O}$ et al. Treatment of acute antibody-mediated rejection. Contrib Nephrol 2017;190:156-167.

3. Kidney Disease: Improving Global Outcomes (KDIGO) Transplant Work Group. KDIGO clinical practice guideline for the care of kidney transplant recipients. Am J Transplant. 2009;9 Suppl 3:S1-S155.

4. Schinstock CA, Mannon RB, Budde K, et al. Recommended Treatment for Antibody-mediated Rejection After Kidney Transplantation: The 2019 Expert Consensus From the Transplantion Society Working Group. Transplantation 2020;104(5):911-922.

5. Swanson J, Schmitz D, and Chung K. How to practice evidence-based medicine. Plast Reconstr Surg 2010 Jul;126(1):286-294.

6. Cooper JE. Evaluation and Treatment of Acute Rejection in Kidney Allografts. Clin J Am Soc Nephrol. 2020;15(3):430-438

7. Hass M, Loupy A, Lefaucheur C et al. The Banff 2017 Kidney Meeting Report: Revised diagnostic criteria for chronic active $\mathrm{T}$ cell-mediated rejection, antibody-mediated rejection, and prospects for integrative endpoints for next-generation clinical trials. Am J Transplant 2018;18(2):293-307.

8. Loupy A, Haas M, Roufosse C, et al. The Banff 2019 Kidney Meeting Report (I): Updates on and clarification of criteria for $\mathrm{T}$ cell- and antibody-mediated rejection. Am \& Transplant. 2020;20(9):2318-2331

9. Prevention and treatment of antibody-mediated rejection of the renal allograft. Available at Uptodate. Accessed Apr 4, 2020.

10. Adebiyi $\mathrm{OO}$, Gralla J, Klem $\mathrm{P}$, et al. Clinical significance of pretransplant donor-specific antibodies in the setting of negative cell-based flow cytometry crossmatching in kidney transplant recipients. Am J Transplant 2016;16:3458-3467.

11. Senev A, Coemans M, Lerut $E$ et al. Histological picture of antibody-mediated rejection without donor-specific anti-HLA antibodies: clinical presentation and implications for outcome. Am J Transplant 2019;19:763-780.

12. Zhang R. Donor-specific antibodies in kidney transplant recipients. Clin I Am Soc Nephrol 2018;13:182-192.

13. Loupy A and Lefaucheur C. Antibody mediated rejection of solid-organ allografts. N Eng/ J Med 2018 20;379(12):1150-1160.

14. Senev A, Coemans M, Lerut E et al. Histological picture of antibody-mediated rejection without donor-specific anti-HLA antibodies: clinical presentation and implications for outcome. Am J Transplant 2019;19(3):763-780.

15. Roberts $D$, Jiang $S$ and Chadban $S$. The treatment of acute antibody-mediated rejection in kidney transplant recipients - a systematic review. Transplantation. 2012; 94(8):775-782.

16. Wan SS, Ying TD, Wyburn K et al. The Treatment of antibody-mediated rejection in kidney transplantation: an updated systematic review and meta-analysis. Transplantation 2018;102(4):557568

17. Velidedeoglu E, Cavaillé-Coll MW, Bala S, et al. Summary of 2017 FDA public workshop: antibodymediated rejection in kidney transplantation. Transplantation 2018;102:e257-e264.

18. Xiea P, Tao M, Peng K, et al. Plasmapheresis therapy in kidney transplant rejection. Blood Purif 2019;47:73-84

19. Levine MH, Abt PL. Treatment options and strategies for antibody mediated rejection after renal transplantation. Semin Immunol 2012;24(2):136-142.

20. Jordan C, Tyan D, Stablein D, et al. Evaluation of intravenous immunoglobulin as an agent to lower allosensitization and improve transplantation in highly sensitized adult patients with endstage renal disease: report of the NIH IG02 Trial. J Am Soc Nephrol 2004;15:3256-3262. 
21. Kasiske BL, Zeier MG, Chapman JR, et al. Kidney disease: improving global outcomes. KDIGO clinical practice guideline for the care of kidney transplant recipients: a summary. Kidney Int 2010;77:299-311.

22. Becker $Y$, Becker B, Pirsh J et al. Rituximab as treatment for refractory kidney transplant rejection. Am J Transplant 2004;4:996-1001

23. Faguer $\mathrm{S}$, Kamar $\mathrm{N}$, Frugier $\mathrm{C}$ et al. Rituximab therapy for acute humoral rejection after kidney transplantation. Transplantation 2007;83:1277-1280.

24. Lefaucheur C, Nochy D, Andrade J et al. Comparison of combination plasmapheresis/IVIg/Anti-CD2O versus High-dose IVIg in the treatment of antibody-mediated rejection. Am J Transplant 2009;9:1099-1107.

25. Sautenet B, Blancho G, Buchler M et al. One year results of the effects of Rituximab on acute antibody-mediated rejection in renal transplantation: RITUX ERAH, a multicenter double blind randomized placebo-controlled trial. Transplantation 2016; 100(2):391-399.

26. Bailly $E$, Ville $S$, Blancho $G$, et al. An extension of the RITUX-ERAH study, multicenter randomized clinical trial comparing rituximab to placebo in acute antibody-mediated rejection after renal transplantation. Transp/ Int. 2020;33(7):786-795.

27. Eskandary $F$, Regele $H$, Baumann $L$ et al. A randomized trial of bortezomib in late antibodymediated kidney transplant rejection. J Am Soc Nephrol 2018;29(2):591-605.

28. Walsh R, Alloway R, Girnita A et al. Proteasome inhibitor-based therapy for antibody-mediated rejection. Kidney International 2012;81:1067-1074.

29. Cornell LD, Schinstock CA, Gandhi MJ et al. Positive crossmatch kidney transplant recipients treated with eculizumab: outcomes beyond 1 year. Am J Transplant 2015;15(5):1293.

30. Burbach M, Suberbielle $C$, Brochériou I et al. Report of the inefficacy of eculizumab in two cases of severe antibody-mediated rejection of renal grafts. Transplantation 2014 Nov;98(10):1056-1059.

31. Lefaucheur C, Viglietti D, Hidalgo L, et al. Complement-activating anti-HLA antibodies in kidney transplantation: allograft gene expression profiling and response to treatment. J Am Soc Nephrol 2018;29:620-635.

32. Kulkami S, Kirkiles-Smith NC, Deng YH et al. Eculizumab therapy for chronic antibody-mediated injury in kidney transplant recipients: a pilot randomized controlled trial. Am J Transplantation 2017;17(3):682

33. Elias M, Nnang-Obada E, Beaudreuil S, et al. Complement inhibition is efficient in early but not late antibody-mediated rejection after kidney transplantation. International Journal of Renal Diseases and Therapy. 2018;1:1-7.

34. Choi J, Aubert O, Loupy A et al. Assessment of tocilizumab (Anti-Interleukin-6 receptor monoclonal) as a potential treatment for chronic antibody-mediated rejection and transplant glomerulopathy in HLA-sensitized renal allograft recipients. Am J Transplantation. 2017;17:2381-2389.

35. Shin BH, Everly MJ, Zhang $\mathrm{H}$, et al. Impact of Tocilizumab (Anti-IL-6R) Treatment on Immunoglobulins and Anti-HLA Antibodies in Kidney Transplant Patients With Chronic Antibody-mediated Rejection. Transplantation. 2020;104(4):856-863.
36. Pottebaum A, PharmD BCPS, Venkatachalam K et al. Efficacy and safety of tocilizumab in the treatment of acute active antibody-mediated rejection in kidney transplant recipients. Transplantation Direct 2020;6:e543.

37. Eskandary F, Dürr M, Budde $K$ et al. Clazakizumab in late antibody-mediated rejection: study protocol of a randomized controlled pilot trial. Trials 2019 11;20(1):37.

38. Vo A, Zeevi A, Choi J et al. A phase I/II placebo-controlled trial of C1-Inhibitor for prevention of antibody-mediated rejection in HLA sensitized patients. Transplantation 2015;99(2):299-308.

39. Montgomery R, Orandi B, Racussen L et al. Plasma-derived C1 Esterase inhibitor for acute antibody mediated rejection following kidney transplantation: results of a randomized double-blind placebo-controlled pilot study. American Journal of Transplantation 2016;16:3468-3478.

40. Viglietti D, Gosset C, Loupy A et al. C1 inhibitor in acute antibody-mediated rejection nonresponsive to conventional therapy in kidney transplant recipients: a pilot study. Am J Transplant 2016;16(5):1596-603.

41. Jordan $\mathrm{S}$, Lorant $\mathrm{T}$, Choi $\mathrm{M}$ et al. IgG Endopeptidase in highly sensitized patients undergoing transplantation. N Engl J Med 2017;377:422-453.

42. Lonze BE, Tatapudi VS, Weldon EP et al. IdeS (Imlifidase): a novel agent that cleaves human IgG and permits successful kidney transplantation across hgh-strength donor-specific antibody. Ann Surg 2018;268(3):488-496.

43. An efficacy and safety of Imlifidase in treatment of antibody mediated rejection in Kidney transplant patients. NCT03897205

44. Konstantin D, Johannes K, Guido A G et al. CD38 antibody daratumumab for the treatment of chronic active antibody-mediated kidney allograft rejection. Transplantation 2020. Publish Ahead of Print.

45. Kumar D, Raynaud M, Chang J, et al. Impact of belatacept conversion on renal function, histology and gene expression in kidney transplant patients with chronic active antibody-mediated rejection. Transplantation 2020. [published online ahead of print, 2020 Apr 17].

46. Jain D, Rajab A, Young JS, et al. Reversing donor-specific antibody responses and antibodymediated rejection with bortezomib and belatacept in mice and kidney transplant recipients [published online ahead of print, 2020 Apr 3]. Am J Transplant. 2020;10.1111/ajt.15881.

\section{Correspondence to:}

Maria do Mar Menezes, MD

Department of Nephrology, Centro Hospitalar Universitário Lisboa Central

Rua da Beneficência 8, 1069-166 Lisbon, Portugal.

E-mail: mariamarmenezes@gmail.com 